\title{
Tuberculosis en Chile: ¿Podemos retomar el camino hacia la eliminación?
}

TANIA HERRERA M.*

Tuberculosis in Chile: are we able to take the road to eliminate this disease?

The World Health Organization established eight guidelines for a new framework in countries with low incidence of tuberculosis (less than 100 cases per million population) to eliminate the disease. Although Chile does not belong to this group since its incidence rate is still 13 cases per 100,000, it is feasible to work in this new framework thanks to the strengths that present the national program. This article analyzes the Chilean situation regarding these guidelines, and determined that most of them have significant progress, but in those related to funding of public health activities and in the development of strategies with specific risk groups some still fail to go forward.

Key words: Tuberculosis, low incidence countries, elimination.

\section{Resumen}

La Organización Mundial de la Salud estableció ocho lineamientos para un nuevo marco de acción en los países con baja incidencia de tuberculosis (menos de 100 casos por millón de habitantes) con miras a eliminar la enfermedad como problema de Salud Pública. Si bien Chile no pertenece a este grupo ya que su tasa de incidencia es aún de 13 casos por 100.000 habitantes, es factible trabajar en este nuevo marco gracias a las fortalezas que presenta el programa nacional. En este artículo se analiza la situación chilena en cuanto a estos lineamientos, determinándose que en la mayoría de ellos se cuenta con avances importantes, pero que en aquellos relacionados con el financiamiento de las acciones desde el punto de vista de Salud Pública y el desarrollo de estrategias especificas con los grupos de riesgo, aún queda camino por avanzar.

Palabras clave: Tuberculosis, paises de baja incidencia, eliminación.

\section{Introducción}

La Organización Mundial de la Salud (OMS) presentó en junio de 2014 un nuevo marco para eliminar la tuberculosis en aquellos países con menos de 100 casos por millón de habitantes, con miras de lograr una incidencia de menos de 1 caso por millón de habitantes para el año 2050 . Treinta y tres son los países que constituyen el grupo al que apunta esta nueva estrategia, siete de los cuales pertenecen a la región de las Américas: Bahamas, Canadá, Costa Rica, Cuba, Estados Unidos de América, Jamaica, y Puerto Rico ${ }^{1}$.

Chile aún no está en este selecto grupo de países debido a que el enlentecimiento de la curva de descenso de la incidencia de tuberculosis (TB) producido en la última década, no ha permitido lograr reducir esta tasa a menos de 10 casos por 100.000 habitantes (100 por millón), a pesar de que ésta era la meta establecida para el año 2010 en los Objetivos Sanitarios 2000-2010². En la actualidad, la tasa de incidencia de Chile contando sólo los casos nuevos ${ }^{1 a}$ (decimales más, decimales menos) está estancada en 13 por 100.000 habitantes desde hace 5 años ${ }^{3}$.

Sin embargo, Chile sí cuenta con los elementos básicos que posibilitarían aplicar las estrategias

${ }^{1 \mathrm{a}} \mathrm{La}$ incidencia, para efectos de comparación internacional, suma a los casos nuevos los casos de recaídas.

* Médico, Directora del Programa de Control y Eliminación de la Tuberculosis-PROCET. Ministerio de Salud. 
definidas en este nuevo marco, ya que posee entre otros, un Programa Nacional de Control de la Tuberculosis de larga data, cobertura universal de diagnóstico y tratamiento, una red de laboratorios organizada y una buen registro de información que permiten mantener un conocimiento acabado sobre la situación ${ }^{4}$. Además, a contar de junio de 2014 se ha actualizado la Norma Técnica del Programa, lo que busca reforzar las actividades de prevención, diagnóstico y tratamiento de la tuberculosis que se desarrollan en el país.

El presente artículo realiza un análisis de la situación actual del Programa de Tuberculosis en Chile en relación a las intervenciones fundamentales que la OMS ha definido como claves para los países con baja carga de TB y que posibilitarían el avance hacia la eliminación de la enfermedad como problema de Salud Pública.

\section{El marco de la Organización Mundial de la Salud}

Ocho son las intervenciones fundamentales definidas por la OMS a desarrollar en los países con baja incidencia de TB para alcanzar la eliminación de la enfermedad ${ }^{1}$ :

1. Garantizar el financiamiento y la rectoría para planificar y ofrecer servicios de calidad.

2. Abordar los grupos más vulnerables e inaccesibles.

3. Abordar las necesidades especiales de los migrantes y cuestiones transfronterizas.

4. Llevar a cabo pruebas de detección de tuberculosis activa y de infección de tuberculosis latente en grupos de alto riesgo y ofrecer un tratamiento adecuado.

5. Mejorar la prevención y atención de la tuberculosis multirresistente.

6. Garantizar la vigilancia continuada y la evaluación y supervisión del programa.

7. Invertir en investigación y en nuevos instrumentos.

8. Apoyar el control mundial de la tuberculosis.

1. El financiamiento del Programa de Tuberculosis en Chile pasa por una situación compleja desde hace cinco años. Durante el año 2008 y 2009 los presupuestos disponibles para realizar las actividades programadas desde el nivel central se redujeron drásticamente de más de 600.000 USD a sólo 95.000 USD $^{2 b}$, situación que a la fecha no ha podido revertirse. En teoría,

${ }^{2 b}$ Cifras calculadas con un cambio de 600 pesos chilenos por dólar americano. el financiamiento de las actividades debían ser asumidas por los propios Servicios de Salud, a través de sus presupuestos históricos y sin un reforzamiento presupuestario adicional ${ }^{6}$. En la actualidad, el financiamiento para los medicamentos antituberculosos de primera y segunda línea, los exámenes de diagnóstico bacteriológico y exámenes complementarios básicos, y la atención de los enfermos y de sus contactos en los distintos niveles del sistema de salud chileno ha sido cubierto por los Servicios de Salud. Sin embargo, existen dificultades para reforzar las actividades de localización de casos, con déficit de personal de laboratorio, falta de insumos e infraestructura inadecuada; dificultades para realizar acciones de supervisión, asesoría y capacitaciones desde los niveles intermedios, por falta de recursos y de miembros de los equipos técnicos; dificultades para realizar actividades de promoción y prevención a nivel poblacional; y en ocasiones, imposibilidad del nivel central para realizar las evaluaciones macro-regionales y las visitas de supervisión ${ }^{7}$.

Si se analiza el trasfondo de la situación del financiamiento, puede observarse que éste se corresponde con la visión del actual sistema de salud chileno, donde lo importante son las prestaciones que se otorgan a nivel individual y enfocadas en el daño, en desmedro de las actividades de salud pública con una perspectiva poblacional. El Programa de Tuberculosis, siendo un programa de Salud Pública, se ha limitado en los últimos años a tratar a los enfermos, muchos de los cuales son diagnosticados en etapas avanzadas de la enfermedad, pasando, por lo tanto, meses manteniendo el contagio. No es de extrañar, entonces, el estancamiento en la disminución de la incidencia que se está observando.

En relación a la rectoría, la existencia de normas técnicas del programa con resolución legal, obligatorias de cumplir en todo el sistema de salud chileno (público y privado), y la existencia de planes y metas definidos tanto en las mismas normas como en la Estrategia Nacional de Salud del país son dos fortalezas con las que cuenta el Programa de Tuberculosis en Chile ${ }^{5,8}$. A esto se agrega que la conducción se lleva desde la Subsecretaría de Salud Pública, no habiendo sufrido la fragmentación a nivel central que ocurrió con otros programas después de la reforma de salud de 2004 .

2. En cuanto a los grupos vulnerables, Chile reconoce la existencia de los llamados grupos de riesgo para tuberculosis. Para el año 2013, un $33,2 \%$ del total de casos de TB pertenecían a alguno de estos grupos (sin considerar a los adultos 
Tabla 1. Porcentaje de casos de tuberculosis que pertenecen a algunos grupos de riesgo. Chile, 2013

\begin{tabular}{|lc|}
\hline Grupo de riesgo & \% \\
\hline Coinfección VIH & 8,7 \\
Extranjeros & 8,4 \\
Alcoholismo/drogadicción & 6,4 \\
Población privada de libertad & 3.9 \\
Diabetes & 3,4 \\
Pueblo indígena & 2,5 \\
Situación de calle & 2,4 \\
\hline
\end{tabular}

Fuente: Informe de situación 2013.

mayores que por sí solos son el $20 \%)^{3}$. La proporción reportada de algunos de estos grupos entre el total de casos de tuberculosis a nivel nacional se muestra en la Tabla 1.

Las cifras muestran un avance en el reporte de los grupos de riesgo a nivel nacional en comparación a los años previos ${ }^{10}$, pero aún deficitaria en algunos grupos como el de personas con diabetes mellitus, para las cuales estudios realizados en Chile señalan prevalencias de al menos un $15 \%{ }^{11}$.

Por otra parte, el reporte de estos grupos no implica el abordaje de los mismos, y en esto el país no ha avanzado sustancialmente. El trabajo intersectorial desarrollado por las SEREMI de Salud aún es escaso, y en los establecimientos de la red asistencial es aún más reducido. En la coinfección TB-VIH existen avances en la coordinación de ambos programas a nivel central y en el desarrollo de un manual de procedimiento conjunto próximo a su publicación, pero el avance a nivel de cada Servicio de Salud es heterogéneo. Para el año 2015 se tiene contemplado el desarrollo de un manual conjunto con gendarmería (Ministerios de Salud y Justicia) para fortalecer las acciones del programa entre las personas privadas de libertad, y existen iniciativas locales y de coordinación a nivel central con el programa de pueblos indígenas, pero todavía en estado incipiente de desarrollo.

3. Respecto a los migrantes y situaciones transfronterizas, actualmente está en funcionamiento el Informe de Traslado Internacional entre Chile, Perú y Bolivia, y las zonas fronterizas de estos tres países realizan reuniones de coordinación en forma sistemática, con lo que el manejo de los casos que se trasladan entre estos países ha mejorado sustancialmente ${ }^{12}$. En el caso de los inmigrantes que permanecen en Chile, si bien a nivel nacional menos del $9 \%$ de los casos de TB se presentan en este grupo, existen algunos Ser- vicios de Salud en los que las proporciones son mucho mayores. El Programa de Tuberculosis está definido como universal, siendo su población objetivo todos los habitantes del territorio nacional, independientemente de su nacionalidad o condición migratoria ${ }^{5}$. Sin embargo, el acceso al sistema de salud puede verse limitado por diversas barreras, especialmente para las personas extranjeras en situación irregular, lo que requiere de un abordaje que va más allá del propio programa $^{13}$. En este sentido, la última disposición de FONASA respecto a la posibilidad de cobertura financiera desde el inicio del trámite de la visa es un avance ${ }^{14}$.

4. El cuarto punto corresponde al diagnóstico y tratamiento de tuberculosis activa y de infección de la tuberculosis latente en grupos de alto riesgo. El diagnóstico de tuberculosis activa tiene cobertura universal y gratuita para las personas viviendo en Chile, y se cuenta con una amplia red de laboratorios que realizan técnicas de baciloscopia y cultivo. La actualización de la norma técnica de 2014 establece que para los grupos de riesgo deben realizarse dos baciloscopias y dos cultivos (en vez de sólo un cultivo que era lo habitual) en sintomáticos respiratorios, lo que busca aumentar la posibilidad de hacer el diagnóstico en estas personas. Además se ha incluido el diagnóstico por PCR (Gene Xpert) como parte de los exámenes en grupos clínicos especiales, como los pacientes con VIH. Se espera contar con esta técnica para todo el país en los próximos años ${ }^{5}$.

Otros avances que se espera poder realizar, y que dependerán del convencimiento de las autoridades de la red asistencial para adquirir los insumos y equipos, son la incorporación de la microscopia de fluorescencia y los cultivos líquidos, buscando acelerar el diagnóstico de tuberculosis.

Referente a la tuberculosis latente, el programa ha establecido el diagnóstico de esta situación y su tratamiento (quimioprofilaxis) en los grupos de mayor riesgo, como menores de 15 años, pacientes con VIH y otras inmunosupresiones, entre las definidas en la Norma ${ }^{5}$. A futuro esta estrategia es probablemente una de las que tendrá que ir ampliándose y mejorándose, para adelantarse a la progresión de infección a enfermedad.

5. La prevención y el manejo de la tuberculosis multidrogorresistente (TB-MDR) es otro de los ámbitos en los que Chile ha avanzado siendo aún pocos los casos que se presentan (19 casos el año 2013) ${ }^{3}$. Además del cambio en los esquemas de tratamiento hacia la simplificación del esquema para casos sensibles y aumento de dosis en la fase intermitente (que pasó de bisemanal a trisemanal), la normativa actual establece que 
todos los casos de TB deben tener un estudio de susceptibilidad inicial a fármacos. Con ello es posible determinar anticipadamente la existencia de resistencias y ajustar en consecuencia el esquema en forma individualizada. A esto se agrega que se lleva una vigilancia centralizada de los casos MDR y de aquellos que presentan resistencia a rifampicina.

6. La vigilancia continuada y la evaluación y supervisión del programa son actividades que se han mantenido e incentivado desde el nivel central. En los últimos tres años se ha hecho al menos una visita de supervisión a cada Servicio de Salud con su respectiva red de establecimientos locales y laboratorios, lo que es una gran fortaleza, considerando que estas visitas permiten observar en terreno la marcha del programa y realizar gestiones regionales y locales para la corrección de las falencias. Además, desde el año 2014 ha comenzado la utilización de una plataforma electrónica para la vigilancia epidemiológica del programa de tuberculosis, actualmente en marcha blanca.

7. Desde el nivel central se realizan todos los años investigaciones operacionales que permiten analizar más profundamente la evolución del programa. Otro tipo de investigaciones desarrolladas por universidades o a través de financiamientos estatales para investigación en salud son ampliamente apoyadas tanto por el Nivel Central como por Seremis y Servicios de salud.

8. Finalmente, en relación a apoyar el control mundial de la tuberculosis, además de la participación activa en las Reuniones Regionales de OPS y Mercosur, Chile cuenta con un Laboratorio Supranacional de Tuberculosis en el Instituto de Salud Pública, el que supervisa y asesora la red de laboratorios de Bolivia, República Dominicana y Colombia, agregándose desde este año Cuba y Uruguay.

\section{Conclusiones}

A pesar que Chile no ha logrado entrar a la fase de pre eliminación de la tuberculosis, definida por la OMS, las estrategias establecidas por esta organización en este nuevo marco de abordaje, pueden ser incorporadas desde ahora en nuestro programa. Es más, varias de ellas ya cuentan con acciones específicas que se están llevando a cabo en el país. Sin embargo, aún queda pendiente un mayor desarrollo en el abordaje de los grupos de riesgo y la recuperación del financiamiento necesario para avanzar tanto en nuevas tecnologías como en el trabajo comunitario e intersectorial. Además es necesario mantener un potente trabajo de los niveles intermedios en cuanto a supervisión y capacitación de las redes. No debe olvidarse que el Programa de Tuberculosis, como programa de Salud Pública, se aleja del modelo actual de atención de salud focalizado en lo curativo e individual, y que los niveles actuales de la endemia obligan a avanzar hacia lo comunitario, lo intersectorial y lo preventivo, si es que queremos romper con el estancamiento de las cifras actuales de incidencia y alcanzar la fase de eliminación de la tuberculosis como problema de Salud Pública.

\section{Bibliografía}

1.- Organización Panamericana de la Salud. La OMS se marca el objetivo de eliminar la tuberculosis en más de 30 países [Internet]. 2014 [cited 2014 Nov 29]. Disponible en: http://www.paho.org/hq/index.php?option $=$ com_content\&view $=$ article $\&$ id $=9751 \&$ Itemid $=1$ 926\&lang=es.

2.- HERRERA T. La situación de la tuberculosis en Chile y los actuales desafíos. Visita de la OPS al programa de control de la tuberculosis de Chile. Rev Chil Enf Respir 2013; 29: 46-9.

3.- Programa Nacional de Control y Eliminación de la Tuberculosis. Tuberculosis: informe de situación Chile 2013 [Internet]. Santiago de Chile; 2014. Disponible en: http://web.minsal.cl/sites/default/files/files/TUBERCULOSIS-INFORME 2013.pdf.

4.- PENAA M C, FARGA C V. El difícil camino del control sanitario de la tuberculosis. Rev Chil Enf Respir 2012; 28: 311-8.

5.- Programa Nacional de Control y Eliminación de la Tuberculosis. Normas técnicas para el control y la eliminación de la tuberculosis [Internet]. 2014. Disponible en: http://web.minsal.cl/sites/default/files/ NORMA_TECNICA_TUBERCULOSIS.pdf.

6.- ZÚÑIGA M. La eliminación de la tuberculosis como problema de Salud Pública: Situación de Chile en el año 2008. Rev Chil Enf Respir 2009; 25: 117-26.

7.- Programa Nacional de Control y Eliminación de la Tuberculosis. Informe de situación 2008-2011. 2012.

8.- Ministerio de Salud. Estrategia Nacional de Salud para el cumplimiento de los Objetivos Sanitarios de la Década 2011-2020.

9.- HERRERA T, SÁNCHEZ S. The absence of stewardship in the Chilean health authority after the 2004 health reform. Medwave [Internet]. Mednet; 2014 nov 26 [cited 2014 nov 29]; 14 (10): e6040-e6040. Disponible en: http://www.mednet.cl/link.cgi/Medwave/Revisiones/ Analisis/6040.

10.- Programa Nacional de Control y Eliminación de la Tuberculosis. Informe de situación 2012. 2013.

11.- HERRERA T, MIRANDA M, CAROLINA O. Asocia- 
ción entre tuberculosis y diabetes mellitus en la Región Metropolitana. 2012; 171-5.

12.- Cooperación Internacional [Internet]. Embajada de Chile en Perú. [cited 2013 sep 18]. Available from: http:// chileabroad.gov.cl/peru/relacion-bilateral/cooperacioninternacional/

13.- DÍAZ J. El caso de las barreras de acceso a las presta- ciones de salud de APS, de los inmigrantes peruanos en la Región Metropolitana de Chile. 2013.

14.- Fondo Nacional de Salud. Inmigrantes podrán acceder a la red pública de salud mientras tramitan su visa [Internet]. 2014. Disponible en: https://www.fonasa.cl/portal_fonasa/site/artic/20141127/pags/20141127131655. html.

Correspondencia a:

Dra. Tania Herrera M.

Directora, Programa de Control y

Eliminación de la Tuberculosis (PROCET)

Ministerio de Salud. Santiago, Chile.

Email: tania.herrera@minsal.cl 